\title{
Location of crossings in the Floquet spectrum of a driven two-level system
}

\author{
C.E. Creffield* \\ Instituto de Ciencia de Materiales (CSIC), Cantoblanco, E-28049, Madrid, Spain \\ (Received 31 October 2002; revised 24 January 2003; published 2 April 2003)
}

\begin{abstract}
The calculation of the Floquet quasi-energies of a system driven by a time-periodic field is an efficient way to understand its dynamics. In particular, the phenomenon of dynamical localization can be related to the presence of close approaches between quasienergies (either crossings or avoided crossings). Here we consider a driven two-level system and study how the locations of crossings in the quasienergy spectrum alter as the field parameters are changed. A perturbational scheme provides a direct connection between the form of the driving field and the quasienergies which is exact in the limit of high frequencies. We first obtain relations for the quasienergies for some common types of applied field in the high-frequency limit, and then show how the locations of the crossings drift as the frequency is reduced. We find a simple empirical formula which describes this drift extremely well in general, and which we conjecture is exact for the specific case of square-wave driving.
\end{abstract}

DOI: 10.1103/PhysRevB.67.165301

PACS number(s): 73.23.-b, 03.65.Xp, 33.80.Be, 03.67.Lx

\section{INTRODUCTION}

The two-level system is a simple model which has been applied to a great variety of physical problems. One application of growing importance is that of quantum computing, ${ }^{1}$ since any quantum two-level system has the potential to act as a quantum bit. For this reason the coherent control of quantum states in these systems has recently become the focus of intense investigation. ${ }^{2,3}$ A concrete example of such a two-level system is provided by a particle tunneling between two potential wells, which can be experimentally realized by confining an electron to a pair of coupled quantum dots. ${ }^{4}$ One method of controlling such a system, without destroying its coherence, is to apply oscillatory electric fields. ${ }^{5}$ Such fields are able to produce the phenomenon known as coherent destruction of tunneling (CDT), in which the tunneling of the particle is suppressed when the parameters of the field are tuned to various "magic" values. As the applied field is timeperiodic, Floquet analysis ${ }^{6}$ has been applied to explain this non-intuitive result, and it has been shown ${ }^{7,8}$ that CDT is closely related to the presence of crossings or avoided crossings in the spectrum of Floquet quasienergies.

The driving field most frequently considered is of sinusoidal form, and studies using CDT as a means of quantum control have generally concentrated on varying either the envelope ${ }^{9}$ or frequency ${ }^{10}$ of a sinusoidal signal. In this work, however, we instead consider the effect of altering the signal's waveform. By using a perturbational method we first show how the waveform can be directly related to the quasienergy spectrum, and give analytic results for sinusoidal, square-wave, and triangular waveforms. These results are precise in the limit of high frequency. As the frequency is reduced, however, the locations of the crossings drift away from these values. This effect is extremely difficult to treat analytically, and such efforts ${ }^{11-13}$ produce complicated results which are difficult to interpret. Empirically, however, we find a simple formula which describes the drifting with good accuracy for many waveforms, and appears to be exact for the case of the square wave. We thus provide a means for predicting the locations of quasienergy crossings for a given driving field in both high and low frequency regimes.

\section{METHOD}

\section{A. Model Hamiltonian}

We consider a charged particle confined to a double quantum-dot system, described by the Hamiltonian

$$
H=\tilde{t}\left(c_{L}^{\dagger} c_{R}+\text { H.c. }\right)+\left[E_{L}(t) n_{L}+E_{R}(t) n_{R}\right],
$$

where the subscript $L / R$ denotes the left/right quantum dot, $c_{j}^{\dagger}$ and $c_{j}$ are creation and annihilation operators for a particle in dot $j$, and $n_{j}=c_{j}^{\dagger} c_{j}$ is the usual number operator. The tunneling between the two dots is described by the hopping parameter $\tilde{t}$, and $E_{j}(t)$ is the electrical potential of the external driving field. Clearly only the potential difference between the two dots is physically of importance, and so we can use the symmetric parametrization,

$$
E_{L}=\frac{E}{2} f(t), \quad E_{R}=-\frac{E}{2} f(t)
$$

where $E$ is the potential of the driving field and $f(t)$ is a $T$-periodic function describing its waveform. Hamiltonian (1) has been written using a basis of localized states, but it may be easily transformed to the standard two-level form via a $\mathrm{SU}(2)$ rotation, yielding the result

$$
H=\frac{\Delta}{2} \sigma_{z}+\frac{E}{2} f(t) \sigma_{x},
$$

where $\sigma_{i}$ are the standard Pauli matrices. In this representation the basis states used are extended states, formed by symmetric and anti-symmetric combinations of the localized states. In the absence of a driving field $(E=0)$ it is clear that the two eigenstates of this Hamiltonian consist of a symmetric ground state, and an excited antisymmetric state. The 
splitting between these two levels is given by $\Delta$, which is related to the interdot tunneling via $\Delta=2 \tilde{t}$.

\section{B. Floquet theory}

As the function $f(t)$ is periodic in time, the Floquet theorem may be used to write solutions of the time-dependent Schrödinger equation as $\psi(t)=\exp \left[-i \epsilon_{j} t\right] \phi_{j}(t)$, where $\phi_{j}(t)$ is a function with the same periodicity as $f(t)$ and is called a Floquet state, and $\epsilon_{j}$ is termed the quasienergy. Although Floquet states have an explicit time dependence, their periodicity means that the dynamics of the system on time scales much larger than the period of the driving field is effectively given only by the quasienergies. In particular, if the two quasi-energies approach degeneracy, the dynamics of the system on this time scale will appear to be frozen, producing the effect of CDT. Consequently, determining the quasienergies provides a simple and direct way of studying the long time-scale behavior of the system, and indicates whether CDT can occur. ${ }^{14}$ In this work we restrict our attention to driving functions which possess the symmetry $f(t)$ $=-f(t+T / 2)$. Imposing this restriction means that Hamiltonian (1) is invariant under the generalized parity operation $x \rightarrow-x, t \rightarrow t+T / 2$, and as a consequence the two Floquet states will also possess this symmetry, one being even and the other being odd. The von Neumann-Wigner theorem ${ }^{15}$ thus allows the two quasienergies to cross as an external parameter, such as the field strength, is varied. Breaking this symmetry by choosing an alternative form for the driving field would mean that the quasi-energies would be forbidden to cross, and thus close approaches between the quasienergies could only consist of avoided crossings.

The Floquet states and their quasienergies may be conveniently obtained from the eigenvalue equation

$$
\left[H(t)-i \frac{\partial}{\partial t}\right] \phi_{j}(t)=\epsilon_{j} \phi_{j}(t) .
$$

To obtain approximate solutions to this equation we follow a perturbation scheme introduced originally by Holthaus ${ }^{16}$ to treat both the two-level system and driven superlattices, and which was generalized recently to also include the effects of inter-particle interactions ${ }^{17}$. In this approach Hamiltonian (1) is divided into two parts: $H_{t}$ which contains the tunneling terms, and $H_{I}$ which holds the electric field terms. We then find the eigensystem of the operator $\mathcal{H}_{I}(t)=H_{I}-i \partial / \partial t$ by working in an extended Hilbert space of time-periodic functions, ${ }^{18}$ and apply the tunneling Hamiltonian as a perturbation. A consequence of dividing the Hamiltonian in this way is that the perturbation theory works well in the highfrequency limit $\omega \gg \widetilde{t}$, but breaks down in the opposite limit when the tunneling provides the dominant energy-scale of the problem. ${ }^{8}$

For the Hamiltonian given in Eq. (1), the problem of finding the eigensystem of $\mathcal{H}_{I}(t)$ simply requires the solution of two uncoupled differential equations:

$$
\left(-\frac{E}{2} f(t)-i \frac{d}{d t}\right) \phi_{+}(t)=\epsilon_{+} \phi_{+}(t)
$$

$$
\left(\frac{E}{2} f(t)-i \frac{d}{d t}\right) \phi_{-}(t)=\epsilon_{-} \phi_{-}(t) .
$$

These can be integrated immediately, giving the solutions:

$$
\phi_{ \pm}(t)=\exp [ \pm i E F(t) / 2] \exp \left[i \epsilon_{ \pm} t\right],
$$

where

$$
F(t)=\int_{0}^{t} f\left(t^{\prime}\right) d t^{\prime} .
$$

The periodicity of the Floquet states clearly requires that $\epsilon_{ \pm}=0 \bmod \omega$. Without loss of generality we can restrict the quasi-energies to lie in the "first Brillouin zone" $(-\omega / 2$ $\leqslant \epsilon<\omega / 2$ ), and thus to lowest order in the perturbation theory they are degenerate and zero. Standard degenerate perturbation theory can now be used to evaluate the firstorder correction to the quasienergies, requiring only that we work in the extended Hilbert space of $T$-periodic functions by defining an appropriate scalar product,

$$
\left\langle\left\langle\phi_{m} \mid \phi_{n}\right\rangle\right\rangle_{T}=\frac{1}{T} \int_{0}^{T}\left\langle\phi_{m}\left(t^{\prime}\right) \mid \phi_{n}\left(t^{\prime}\right)\right\rangle d t^{\prime},
$$

where $\langle\cdot \mid \cdot\rangle$ is the usual scalar product for the spatial component of the wave functions, and $\langle\cdot \mid \cdot\rangle_{T}$ denotes the integration over the compact time coordinate.

As the tunneling component of the Hamiltonian $H_{t}$ is acting as the perturbation, the first-order approximation of the quasienergies is given by the eigenvalues of the perturbing matrix:

$$
\left\langle\left\langle H_{t}\right\rangle\right\rangle_{T}=\left(\begin{array}{cc}
0 & \tilde{t}\left\langle\phi_{-}^{2}\right\rangle_{T} \\
\tilde{t}\left\langle\phi_{+}^{2}\right\rangle_{T} & 0
\end{array}\right) .
$$

Comparing this expression with the original tunneling Hamiltonian [Eq. (1)] reveals that the action of the applied field is to renormalize the tunneling terms by the factors $\left\langle\phi_{ \pm}^{2}\right\rangle_{T}$. As $\phi_{+}$is the complex conjugate of $\phi_{-}$, the quasienergies take the simple form

$$
\epsilon_{ \pm}= \pm \frac{\Delta}{2}\left|\left\langle\phi_{+}^{2}\right\rangle_{T}\right|
$$

where

$$
\left\langle\phi_{+}^{2}\right\rangle_{T}=\frac{1}{T} \int_{0}^{T} \exp [i E F(t)] d t,
$$

and $F(t)$ is defined in Eq. (8). Clearly the quasi-energies can only become degenerate when they are both equal to zero, and we can note from Eq. (10) that this corresponds, as expected, to the destruction of the effective tunneling.

\section{RESULTS}

To obtain the Floquet quasienergies for comparison with the prediction of Eq. (12), the numerical technique described in Ref. 17 was used. This involves evaluating the unitary evolution operator for one period of the field $U(T, 0)$ and 
obtaining its eigenvalues, which are related to the quasienergies via $\lambda_{j}=\exp \left[-i \epsilon_{j} T\right]$. Using this method to obtain the quasienergies, a standard bisection algorithm could then be used to find the location of the quasienergy crossings to a high degree of accuracy.

The dynamical behavior of the system was also examined directly by integrating it over long time-periods, with the particle initially located in the left quantum dot. To quantify to what extent the tunneling between the left and right quantum dots was destroyed, the probability that the particle was in the left quantum $\operatorname{dot}\left[P_{L}(t)\right]$ was measured throughout the time evolution. We denote the minimum value of $P_{L}$ attained during this period to be the "localization," and thus high values of localization correspond to the presence of CDT, while low values reveal that the particle is able to tunnel from one side to the other, and is therefore delocalized.

\section{A. Sinusoidal driving}

We begin with the most familiar case, when the driving field has the form $f(t)=\cos \omega t$. The procedure outlined in Sec. II B can be followed straightforwardly, leading to the result that

$$
\left\langle\phi_{+}^{2}\right\rangle_{T}=\frac{1}{T} \int_{0}^{T} \exp [i E \sin (\omega t) / \omega] d t .
$$

By making use of the standard identity

$$
\exp [i E \sin (\omega t) / \omega]=\sum_{m=-\infty}^{\infty} J_{m}(E / \omega) \exp [i m \omega t]
$$

this expression can be substantially simplified, yielding the final result that $\epsilon_{ \pm}= \pm(\Delta / 2) J_{0}(E / \omega)$. This reproduces the well-known result that for sinusoidal driving CDT occurs when the ratio of the field strength to its frequency is equal to a root of the Bessel function $J_{0}$. In Fig. 1(a) the locations of the quasienergies are shown for a fixed frequency $\omega=8$ as a function of $E / \omega$. It can be seen that the perturbative result works extremely well in this regime (high-frequency). Figure 1(b) shows the localization produced by the field, as defined above. As expected, at the points where the quasienergies cross the tunneling dynamics of the system is blocked, producing sharp spikes in the localization, centered on the crossings.

To investigate how the crossings move away from these points as the driving frequency is reduced, their locations are shown as a function of $1 / \omega$ in Fig. 2(a). In accordance with the von Neumann-Wigner theorem ${ }^{8,15}$ we can readily see that the set of crossings form one-dimensional manifolds. As $\omega$ tends to infinity the crossings occur at the roots of $J_{0}$, as predicted by the perturbation theory, and this remains a good approximation for frequencies as low as $\omega=\Delta$. Below this value, however, the crossings smoothly drift away from these locations, and evolve towards the points $\Delta / \omega=2 n$ (where $n$ is a positive integer), as was seen earlier in Ref. 8. This limiting behavior in the low-frequency regime was also predicted in Ref. 19, where a similar pattern of crossing-drift was observed in an investigation of a related model. The

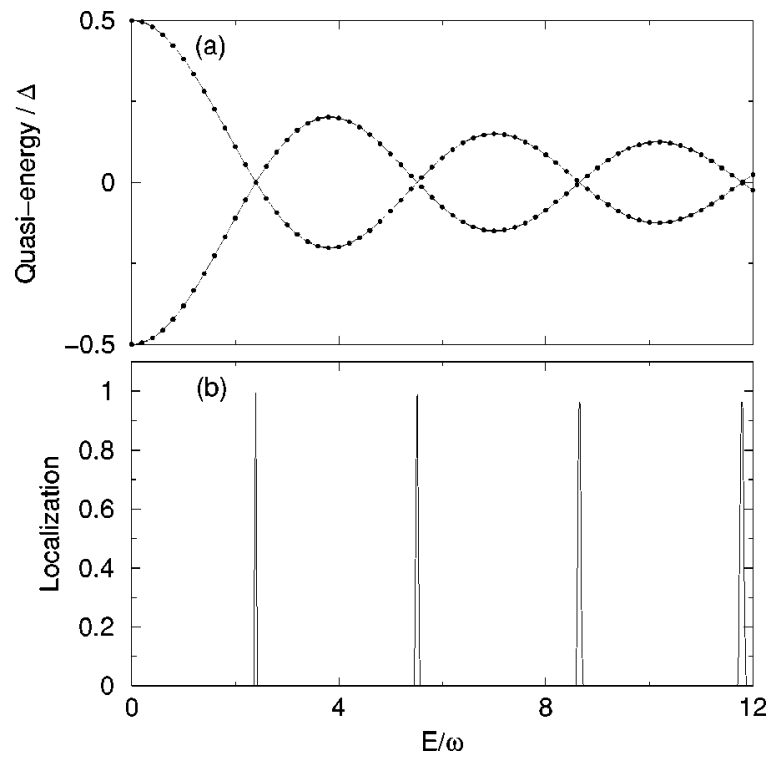

FIG. 1. (a) Quasienergies for a sinusoidal driving field, of frequency $\omega=8$. Circles indicate exact results, lines the perturbative result $\pm(\Delta / 2) J_{0}(E / \omega)$. (b) Localization in the driven system. Spikes in the localization are centered on crossings of the quasienergies.

form of Fig. 2(a) immediately suggests fitting the manifolds of crossings with quadrants of ellipses,

$$
\left(\frac{E / \omega}{y_{n}}\right)^{2}+\left(\frac{\Delta / \omega}{2 n}\right)^{2}=1
$$

where $y_{n}$ is the $n$-th root of $J_{0}(y)$. It can be seen in Fig. 3 that this simple parametrization fits the results extremely well for the first crossing manifold, and that the difference between the exact location of the crossing and the fitting function $\left(E_{\text {fit }} / \omega-E_{\text {exact }} / \omega\right)$ never exceeds a value of 0.02 . The degree of deviation becomes larger as the order of the crossing increases, but nonetheless is only visible in Fig. 2(a) for the fourth and fifth crossing-manifolds.

In Fig. 4 the localization is plotted as $\omega$ is reduced from a high value toward zero, with $E$ set to hold the ratio $E / \omega$ on a crossing manifold. For each point the system was evolved over 200 periods of the driving field to study how effectively the field could maintain a localized state. For the highfrequency regime, $\omega \geqslant \Delta$, the localization is excellent at all the crossings, with less than 0.1 of the particle density leak-

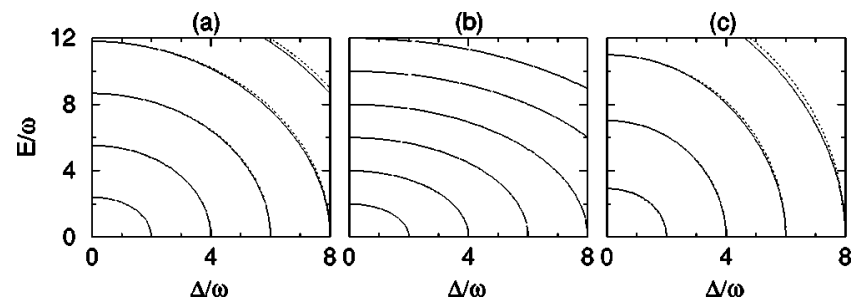

FIG. 2. Location of crossings of quasienergies, in each case the crossings fall on one-dimensional manifolds. (a) Sinusoidal driving. (b) Square-wave driving. (c) Triangular driving. Dotted lines indicate the empirical fitting function [Eq. (15)]. 


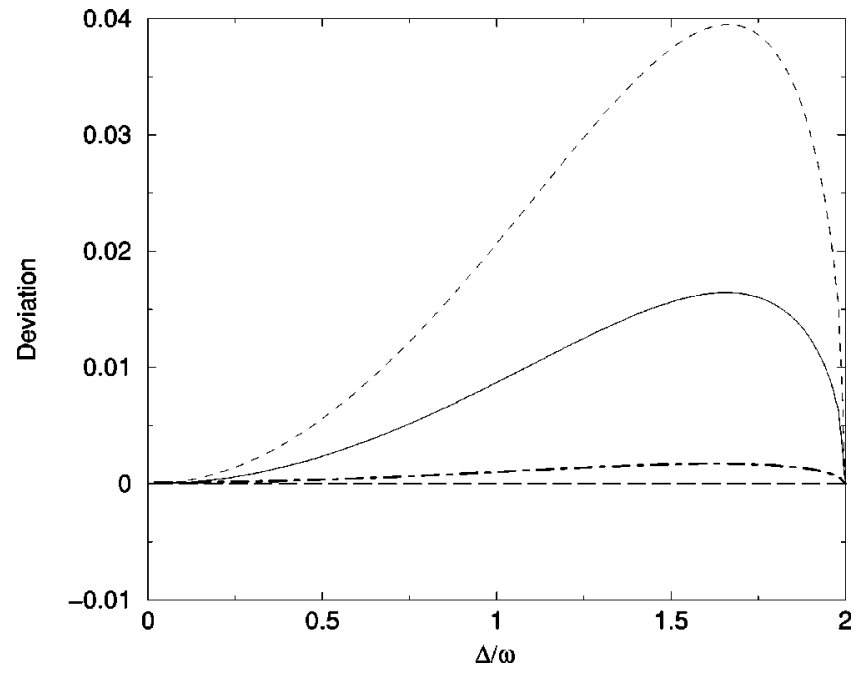

FIG. 3. Deviation of the first crossing manifold from the empirical fitting function. The solid line indicates sinusoidal driving, the dashed line square-wave driving, and the dotted line triangular driving. For the square wave the deviation is smaller than $10^{-7}$. The dot-dashed line gives the deviation for the Fourier expansion of the square wave, truncated at two terms.

ing across to the right-side dot during the time evolution. As can be expected, the high-order crossings, which occur at higher values of $E$, can maintain better levels of localization than the low-order crossings. ${ }^{8}$ This difference becomes more pronounced as the frequency is reduced, and although the localization in all cases decays smoothly to zero, the localization at the higher-order crossings decays much more slowly. For frequencies as low as $\omega=0.4 \Delta$, however, the inhibiting effect of CDT is still evident for all the crossings, indicating that even low-frequency fields may serve a useful role in stabilizing electron-leakage from quantum dot devices.

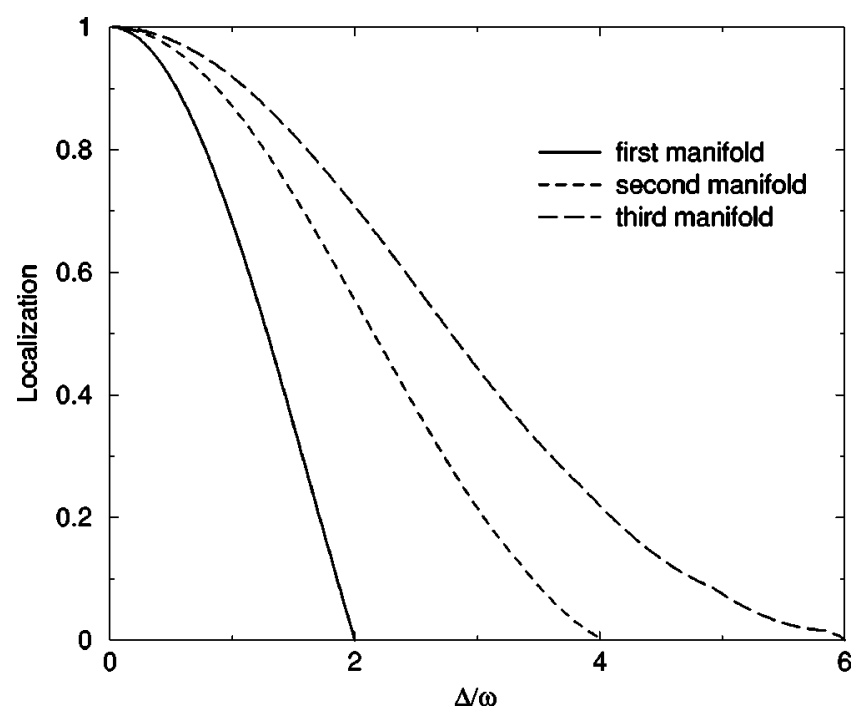

FIG. 4. Localization produced by a sinusoidal field, for $(E, \omega)$ coordinates lying on the first three crossing manifolds.

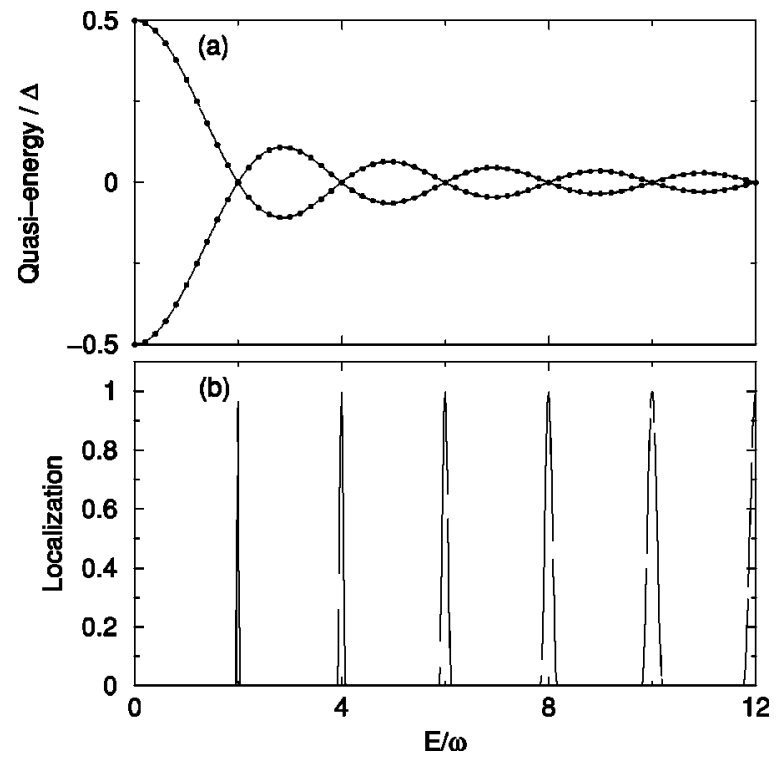

FIG. 5. (a) Quasienergies for a square-wave driving field, of frequency $\omega=8$. Circles indicate exact results, lines the perturbative result [Eq. (16)]. (b) Localization produced by the driving field.

\section{B. Square-wave driving}

Square-wave driving has been considered to a lesser extent than the sinusoidal case, although it is also an easily realizable waveform in experiment. Reference 20 investigated the case of a superlattice driven by a square-wave field, and found that for suitable choices of parameters CDT would indeed occur, while sinusoidal driving of this system could only produce partial CDT. ${ }^{21}$ Recently in Ref. 22 it was shown that in a superlattice CDT can only be produced if the crossings of the quasienergies are equally spaced, which clearly does not occur for sinusoidal driving. For this reason it is of interest to derive the behavior of the quasienergies for square-wave driving to see explicitly how this condition is fulfilled.

We consider the square-wave driving field $f(t)=\Theta(t)$ $-2 \Theta(t-T / 2)$, defined over the interval $0 \leqslant t<T$. The integrations required to obtain the quasienergies may again be done straightforwardly, giving the result that

$$
\epsilon_{ \pm}= \pm \frac{\Delta}{2} \frac{\sin (\pi E / 2 \omega)}{\pi E / 2 \omega} .
$$

From this it is immediately clear that the crossings are equally spaced as required, being given by the condition $E / \omega=2 n$ where $n$ is a positive integer. In Fig. 5(a) the quasienergies obtained for a frequency of $\omega=8$ are shown in comparison with the above result, and it can be clearly seen that the agreement is excellent. Below this figure is plotted the localization produced by the field, and as for the case of sinusoidal driving, the crossings of the quasienergies correspond to sharp spikes in the localization, verifying that CDT is indeed occurring.

In Fig. 2(b) the drifting of the crossings as the frequency is reduced is shown. The behavior is strikingly similar to that observed for sinusoidal driving, and accordingly we use the same functional form [Eq. (15)] to fit the crossing manifolds, 


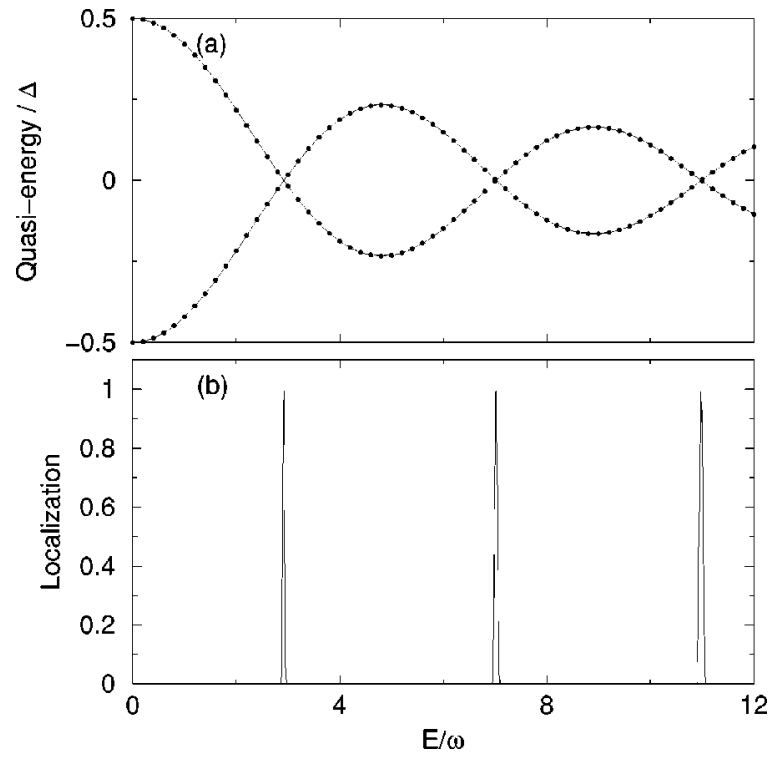

FIG. 6. (a) Quasienergies for a triangular driving field, of frequency $\omega=8$. Circles indicate exact results, lines the perturbative result [Eq. (18)]. (b) Localization produced by the driving field.

with the $y$ intersections now given by $y_{n}=2 n$. The fit is so good that on this plot no differences can be seen between the exact results and the fits. This is corroborated by Fig. 3, where the deviation from the exact result for the lowest manifold can be seen to be negligible in comparison with the sinusoidal case, and within the accuracy of the numerical procedures the fit is identical with the exact result. We therefore conjecture that this fitting is, in fact, exact for the case of square-wave driving. In this plot we also show the result obtained for a bandwidth-limited square-wave, obtained by truncating its Fourier expansion at two terms. We see that the addition of just the second term to the sinusoidal driving already reduces the deviation of the fit from the exact result considerably. Truncating the series at higher points produces steady improvements in the fit, strongly supporting the conjecture that the fit is exact when all terms are included.

\section{Triangular driving}

We now consider another easily obtainable form of driving, the triangular waveform:

$$
f(t)= \begin{cases}1-4 t / T & \text { for } 0 \leqslant t \leqslant T / 2 \\ -3+4 t / T, & T / 2<t \leqslant T\end{cases}
$$

For this case a closed form solution can again be obtained for the behavior of the quasienergies, involving the Fresnel sine and cosine functions, $S(x)$ and $C(x)$. The full expression for the quasienergies is given by

$$
\epsilon_{ \pm}=\frac{\Delta}{\sqrt{2 x}}[\cos (x \pi / 4) C(\sqrt{x / 2})+\sin (x \pi / 4) S(\sqrt{x / 2})],
$$

where $x=E / \omega$. In Fig. 6 it can be seen that this function is indeed an excellent approximation to the true quasi-energies, and that CDT again occurs at the points of quasienergy crossings. The roots of Eq. (18) may be found numerically, yielding the result that the first three crossings occur when $E / \omega=2.92519,7.02525$, and 10.9864. Observing the behavior of the Fresnel functions ${ }^{23}$ reveals that for $x>1$ they both make small amplitude, decaying oscillations about a value of 0.5 , which allows the condition for crossings to be written in the simpler, though approximate, form $\tan (x \pi / 4) \simeq-1$. The crossing condition therefore reduces to the simple result $E / \omega \simeq 4 n-1$, as may be seen from the exact values given above, which becomes increasingly accurate for larger values of $E / \omega$.

In Fig. 2(c) it can be seen that the crossing-manifolds for this form of driving have a similar elliptical form to the previous cases. Using the same fitting function [Eq. (15)] as before, with the $y$ intercepts given by the roots of Eq. (18), gives an accurate description of their behavior, as may be seen in Fig. 3. Although the fit is not as good as for the sinusoidal case, the maximum deviation is still less than 0.04 . As seen previously, the fit is best for the lowest-order manifolds, with small deviations being visible in the higherorder manifolds. Nonetheless, in all cases the fitting function gives an impressively accurate approximation to the true result.

\section{CONCLUSIONS}

In summary, it has been shown how changing the waveform of a periodic driving field can be used to modify the location of the quasienergies of a two-level system. A procedure has been given which relates the waveform explicitly to the quasienergy spectrum, allowing the positions of the quasienergy crossings to be located exactly in the limit of high frequency. For various driving fields, including the cases we consider here, an analytic form can be obtained for the quasienergies, and in other cases they may be obtained numerically with little difficulty. This gives the prospect of designing the waveform to create a desired behavior of the quasienergy spectrum in a direct and straightforward way.

It has also been shown how the positions of the quasienergy crossings drift as the frequency is reduced from the high-frequency limit. For the driving fields considered here, the crossings fall approximately onto elliptical manifolds, and for the case of square-wave driving it appears that this description is exact. We have examined this behavior for many other waveforms, and we conclude that this form of the crossing manifolds is very general. Using the perturbation theory to find the crossings in the high-frequency limit, and then making use of this drifting behavior, allows the positions of the quasienergy crossing to be accurately located in all regimes of driving. This gives more flexibility in experiment, as the high-field regime may either be difficult to attain, or may induce undesirable transitions to higher energy levels, breaking the two-level approximation. Although the degree of localization that the field can maintain is reduced in the low-frequency regime, it can still produce a useful reduction of the leakage from quantum dot devices, and thereby enhance their decoherence time, which has many possible applications to the coherent control of mesoscopic systems. 


\section{ACKNOWLEDGMENT}

This research was supported by the EU through the TMR program "Quantum Electron Transport in the Frequency and
Time Domains." The author thanks Gloria Platero for discussions, and acknowledges the hospitality of the International Institute for Applied Systems Analysis (IIASA) in Vienna, where part of this work was carried out.
*Current address: Dipartimento di Fisica, Università di Roma "La Sapienza," Piazzale Aldo Moro 2, I-00185, Roma, Italy.

${ }^{1}$ C.H. Bennett and D.P. DiVincenzo, Nature (London) 404, 247 (2000).

${ }^{2}$ D. Vion, A. Aassime, A. Cottet, P. Joyez, H. Pothier, C. Urbina, D. Esteve, and M.H. Devoret, Science 296, 886 (2002).

${ }^{3}$ B.E. Cole, J.B. Williams, B.T. King, M.S. Sherwin, and C.R. Stanley, Nature (London) 410, 60 (2001).

${ }^{4}$ R.H. Blick, D. Pfannkuche, R.J. Haug, K. von Klitzing, and K. Eberl, Phys. Rev. Lett. 80, 4032 (1998).

${ }^{5}$ T.H. Oosterkamp, T. Fujisawa, W.G. van der Wiel, K. Ishibashi, R.V. Hijman, S. Tarucha, and L.P. Kouwenhoven, Nature (London) 395, 873 (1998).

${ }^{6}$ Jon H. Shirley, Phys. Rev. 138, B979 (1965).

${ }^{7}$ F. Grossmann, T. Dittrich, P. Jung, and P. Hänggi, Phys. Rev. Lett. 67, 516 (1991).

${ }^{8}$ F. Grossmann and P. Hänggi, Europhys. Lett. 18, 571 (1992).

${ }^{9}$ Martin Holthaus, Phys. Rev. Lett. 69, 1596 (1992).

${ }^{10}$ K. Drese and M. Holthaus, Eur. Phys. J. D 5, 119 (1999).

${ }^{11}$ J.C.A. Barata and W.F. Wreszinski, Phys. Rev. Lett. 84, 2112 (2000)
${ }^{12}$ Marco Frasca, Phys. Rev. A 60, 573 (1999).

${ }^{13}$ V. Delgado and J.M. Llorente, J. Phys. B 33, 5403 (2000).

${ }^{14}$ It should be noted, however, that although quasienergy degeneracy is necessary to produce CDT it is not sufficient. For example, if the Floquet states themselves have a large amplitude of oscillation, the particle will not be localized on short time scales, and thus CDT will not occur.

${ }^{15}$ J. von Neumann and E. Wigner, Phys. Z 30, 467 (1929).

${ }^{16}$ M. Holthaus, Z. Phys. B: Condens. Matter 89, 251 (1992).

${ }^{17}$ C.E. Creffield and G. Platero, Phys. Rev. B 65, 113304 (2002).

${ }^{18}$ Hideo Sambe, Phys. Rev. A 7, 2203 (1973).

${ }^{19}$ J.M. Villas-Bôas, Wei Zhang, Sergio E. Ulloa, P.H. Rivera, and Nelson Studart, Phys. Rev. B 66, 085325 (2002).

${ }^{20}$ Ming Jun Zhu, Xian-Geng Zhao, and Qian Niu, J. Phys.: Condens. Matter 11, 4527 (1999).

${ }^{21}$ Xian-Geng Zhao, J. Phys.: Condens. Matter 6, 4527 (1994).

${ }^{22}$ M.M. Dignam and C. Martijn de Sterke, Phys. Rev. Lett. 88, 046806 (2002)

${ }^{23}$ Handbook of Mathematical Functions, edited by M. Abramowitz and I.A. Stegun (Dover, New York, 1972). 